Military Technical College Kobry El-Kobbah, Cairo, Egypt

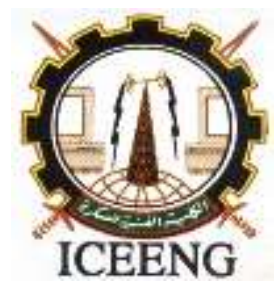

$11^{\text {th }}$ International Conference on Electrical Engineering ICEENG 2018

\title{
PERFORMANCE MATRICES IN MV EGYPTIAN DISTRIBUTION SYSTEMS
}

Dina M.Said ${ }^{*}$, Kamelia Youssef ${ }^{* *}$, Salma Hussien ${ }^{* * *}$ and Hatem Waheed ${ }^{* * \star}$

\section{ABSTRACT}

Distribution power systems can be very complex, integrating many different types of components; circuit breakers, fuses, cables, overhead lines, transformers and auxiliaries which are used to provide electricity to a number of consumers with varying requirements. Each individual component has its own characteristics, including its reliability.

The power supply reliability will be measured, monitored, evaluated by using some number of performance matrices, mainly for: i) frequency of outages (SAIFI, ASIFI) and ii) duration of outages (SAIDI, ASIDI, and CAIDI). These matrices are based on sustained outages. Sustained interruptions are those that last more than five minutes [1].

This paper presents and analyzes the statistical performance matrices for the previous 10 years for the distribution system in Egypt and how it can be used to help electricity Distribution Companies (EDCs) to improve the reliability of their networks' electric components.

\section{KEY WORDS}

Reliability, Duration, Outages, Frequency, Load.

* Electric Engineer, Egypt ERA, Cairo, Egypt.

** Senior Electric Engineer, Egypt ERA, Cairo, Egypt.

*** Energy Efficiency Consultant, Egypt ERA, Cairo, Egypt.

${ }^{* * * *}$ Executive Manager, Egypt ERA, Cairo, Egypt. 


\section{Introduction}

\section{Egyptian Electric Utility:}

The Egyptian Electric Utility and Consumer Protection Regulatory Agency (Egypt ERA) is regulating all matters related to electric utility. Egyptian Electricity Holding Company (EEHC) has 16 affiliated companies (6 Generation, 9 Distribution and the Egyptian Electricity Transmission Company). Distribution Facilities in Egypt are operated by 9 Electricity Distribution companies (EDCs). The EDCs are operating within specific geographical zones. They are responsible for power supply and grid operation within its specific geographic zone (medium voltage (MV) and low voltage $(\mathrm{LV}))$.

Measures of reliability matrices, such as the frequency and duration of electric power interruptions have been reported by Egypt ERA since 2006/2007. The report centered in: SAIFI, SAIDI, CAIDI, ASIFI and ASIDI. These metrics can be used to develop a comprehensive assessment of reliability nationwide.

\section{MV distribution systems:}

In Egypt, a typical distribution system is:

- Ring grid

- $22 \mathrm{kV}, 11 \mathrm{kV}$ lines (cables, overhead, distributors)

- Distribution transformers (22/0.4 kV, 11/0.4 kV) 3-phases.

- distributed load

All these components contribute to reliability.

The reasons for outages are either:

- Independent of weather conditions; such as trees, squirrels.

or

- Strongly dependent on weather conditions, such as lightning and wind.

In the Egyptian distribution system, a sustained network interruption time is 5 minutes for MV networks, which matches with the IEEE 1366 standard. The sustained interruptions can be divided into the planned and unplanned components. The planned interruptions are described as controllable events, but the unplanned (fault) ones as uncontrollable event.

Statistical data point to, within all electric power systems, a medium voltage (MV) cable distribution networks have a dominant influence on the reliability of supply end users $(\approx 80 \%)$ in total reliability indices of power system.

Customers expect the electric grid to be reliable, the business sector wants the electric grid to stop damaging devices and equipment, everyone wants pure electricity and less fuel use.

EDCs works on fulfilling the consumers' needs by:

- Decreasing number of outages

- Quick restoration time 
- Rapid response to complainers.

- Safe operation of electric devices and equipment.

\section{STANDARD RELIABILITY MATRICES}

A wide variety of matrices has been defined to measure interruptions of electric power system [1]. Reality matrices should include at minimum: SAIFI, CAIDI (as a weight of the interruption based on number of customers), ASIFI, ASIDI (as based on load).

For entire network and include MV (22 kV, $11 \mathrm{kV})$ interruptions. Table 1. summarizes recommended reliability matrices.

\section{EGYPT ERA DATABASE}

The performance matrices have been calculated, according to Egypt ERA software, based on the huge data provided by EDCs. Historical Performance matrices based on sustained outages during 10 years interval for EDCs are calculated, observed and analyzed.

\section{Continuous Improvement}

After observation for matrices during interval 2006/2007 to 2010/2011, the strategies were implemented according to Table 2 in addition to matching with improving power quality for electrical parameters.

Historical performance matrices based on sustained outages during 10 years interval for MV EDCs networks are presented in Figs 1, 2 and 3.

Fig.4 represents five-year moving matrices (SAIFI \& CAIDI) related to historical performance data

The graph in Fig. 4 is broken into quadrants using a cutoff for SAIFI of 1.0 for "few outages" versus" many outages" and a cutoff of just over 100 CAIDI minutes for "short outages" versus" long outages". The graph indicates that most EDCs fall into the quartile $(A)$ of few outages.

The observation of results in Figs 1, 2, 3 and Fig. 4 during last 5 years is presented in Table 3.

Also historical performance matrices based on load during years 2014/2015 \& 2015/2016 for EDCs are calculated for planned and unplanned outages and are presented in Tables 4 and 5. Total performance matrices as based on load are shown in Table 6.

Fig. 5 presents two -year moving matrices related to historical performance data in Table 4. The graph in Fig 5 indicates that most EDCs fall under $150 \mathrm{~min} /$ outages.

The comparison between Fig 4 and Fig 5 pointed to the two calculations methods for reliability matrices, one based on customers and other based on load, are given far 
figures, independent and each EDCs should choose one of them to build its historical reliability matrices.

\section{CONCLUSION}

The evaluation of reliability matrices in MV electric distribution systems in Egypt has been presented by two methods: one based on number of customers and the other based on load.

The reliability analysis tools allow a detailed analysis of the results after calculating, monitoring and measuring the performance matrices. Besides overall values for each power system of EDC, the results for different load district can be analyzed individually. This helps supports and includes the important information to EDCs and weak load districts in the system can be detected and improvement measures taken. Finally, reliability matrices analysis is a powerful tool in addressing the requirements of the new electric distribution component market. Also performance matrices and reporting should be continuously refined.

\section{Tables}

Table 1. Recommended reliability matrices

\begin{tabular}{|c|c|}
\hline \multirow{3}{*}{ SAIFI } & System Average Interruption Frequency Index \\
\hline & Measures system wide outage frequency for sustained outages. \\
\hline & $\begin{array}{c}\text { Equal ( } \sum \text { total number of customers interrupted) / } \\
\text { (Total number of customers served) }\end{array}$ \\
\hline \multirow{3}{*}{ SAIDI } & System Average Interruption Duration Index \\
\hline & Measures annual system-wide outage duration for sustained outages) \\
\hline & $\begin{array}{l}\text { Equal, }\left(\sum \text { customer minutes of interruption }\right) / \\
\text { (Total number of customers served })\end{array}$ \\
\hline \multirow{3}{*}{ CAIDI } & Customer Average Interruption Duration Index \\
\hline & Measures average duration of sustained outage per customer. \\
\hline & Equal, ( SAIDI, SAIFI) \\
\hline \multirow{3}{*}{ ASIFI } & Average System Interruption Frequency Index \\
\hline & $\begin{array}{l}\text { Measures distribution performance in area that serve relatively few } \\
\text { customers ( have large connections of load ) }\end{array}$ \\
\hline & $\begin{array}{c}\text { Equal ( } \sum \text { total connected kVA of load interrupted) / } \\
\text { ( Total connected kVA served) }\end{array}$ \\
\hline \multirow{3}{*}{ ASIDI } & Average System Interruption Duration Index \\
\hline & Measures outage duration based on load \\
\hline & $\begin{array}{l}\text { Equal ( } \sum \text { connected kVA duration of load interrupted) / } \\
\text { ( Total connected kVA served) }\end{array}$ \\
\hline
\end{tabular}


Table 2. Strategies for improving matrices

\begin{tabular}{|c|c|}
\hline $\begin{array}{l}\text { Reduction } \\
\text { of the } \\
\text { number of } \\
\text { outages }\end{array}$ & $\begin{array}{l}\text { - Preventive maintenance } \\
\text { - replacement the bad components } \\
\text { - use isolated overhead lines } \\
\text { - protection against animal } \\
\text { - avoid overloading of transformer and feeders } \\
\text { - employ better quality devices } \\
\text { - planned program of checks } \\
\text { - prioritization and implementation }\end{array}$ \\
\hline $\begin{array}{l}\text { Reduction } \\
\text { of times of } \\
\text { outages }\end{array}$ & $\begin{array}{l}\text { - reconfiguration of grid } \\
\text { - coordination of protective relay settings } \\
\text { - fault current protection } \\
\text { - automation control of distribution network }\end{array}$ \\
\hline
\end{tabular}

Table 3. Observation during $2011 / 2012$ to $2015 / 2016$

\begin{tabular}{|c|c|c|c|}
\hline EDCs & SAIFI & SAIDI & CAIDI \\
\hline EDC1 & $\mathrm{I}$ & $\mathrm{I}$ & $\mathrm{A}$ \\
\hline EDC2 & $\mathrm{I}$ & $\mathrm{I}$ & $\mathrm{I}$ \\
\hline EDC3 & $\mathrm{O}$ & $\mathrm{O}$ & $\mathrm{I}$ \\
\hline EDC4 & $\mathrm{A}$ & $\mathrm{O}$ & $\mathrm{O}$ \\
\hline EDC5 & $\mathrm{I}$ & $\mathrm{I}$ & $\mathrm{I}$ \\
\hline EDC6 & $\mathrm{I}$ & $\mathrm{A}$ & $\mathrm{B}$ \\
\hline EDC7 & $\mathrm{I}$ & $\mathrm{O}$ & $\mathrm{B}$ \\
\hline EDC8 & $\mathrm{I}$ & $\mathrm{O}$ & $\mathrm{O}$ \\
\hline EDC9 & $\mathrm{I}$ & $\mathrm{O}$ & $\mathrm{B}$ \\
\hline
\end{tabular}

* I = improvement

$A$ = approximately improved

$\mathrm{O}=$ oscillating

$\mathrm{B}=\mathrm{Bad}$

Table 4. Development of load-based index ASIFI for planned and unplanned outages (inter./customer)

\begin{tabular}{|c|c|c|c|c|}
\hline \multirow{2}{*}{} & \multicolumn{2}{|c|}{ Planned } & \multicolumn{2}{c|}{ Unplanned } \\
\cline { 2 - 5 } & $2014 / 2015$ & $2015 / 2016$ & $2014 / 2015$ & $2015 / 2016$ \\
\hline EDC1 & 2.15 & 1.36 & 1.36 & 1.31 \\
\hline EDC2 & 1.608 & 1.41 & 0.374 & 0.347 \\
\hline EDC3 & 1.109 & 0.968 & 0.721 & 0.828 \\
\hline EDC4 & 0.923 & 0.786 & 1.771 & 1.802 \\
\hline EDC5 & 1.06 & 1.05 & 2.74 & 2.5 \\
\hline EDC6 & 0.26 & 0.25 & 0.37 & 0.36 \\
\hline EDC7 & 3.086 & 2.973 & 3.496 & 2.7 \\
\hline EDC8 & 10.539 & 3.7 & 2.32 & 1.78 \\
\hline EDC9 & 0.7 & 0.59 & 0.58 & 1.22 \\
\hline
\end{tabular}


Table 5.Development of load-based index ASIDI for planned and unplanned outages ( inter./customer)

\begin{tabular}{|c|c|c|c|c|}
\hline \multirow{2}{*}{} & \multicolumn{2}{|c|}{ Planned } & \multicolumn{2}{c|}{ Unplanned } \\
\cline { 2 - 5 } & $2014 / 2015$ & $2015 / 2016$ & $2014 / 2015$ & $2015 / 2016$ \\
\hline EDC1 & 366.15 & 238.15 & 132.23 & 116.22 \\
\hline EDC2 & 203.2 & 185.889 & 5.43 & 50.038 \\
\hline EDC3 & 125.4 & 114.8 & 75 & 72 \\
\hline EDC4 & 85.835 & 72.989 & 79.787 & 78.189 \\
\hline EDC5 & 88.05 & 86.1 & 89.34 & 80.42 \\
\hline EDC6 & 9.88 & 9.59 & 9.7 & 9.5 \\
\hline EDC7 & 501.8 & 468.2 & 365.3 & 297.1 \\
\hline EDC8 & 1246.1 & 480.7 & 239.2 & 266.69 \\
\hline EDC9 & 58.12 & 54.23 & 115.51 & 240.63 \\
\hline
\end{tabular}

Table 6.Development of performance matrices as based on load for 9 EDCs

\begin{tabular}{|c|c|c|c|c|c|c|}
\hline \multirow{2}{*}{} & \multicolumn{2}{|c|}{ ASIFI } & \multicolumn{2}{c|}{ ASIDI } & \multicolumn{2}{c|}{ Min/ outage } \\
\cline { 2 - 7 } & $2014 / 2015$ & $2015 / 2016$ & $2014 / 2015$ & $2015 / 2016$ & $2014 / 2015$ & $2015 / 2016$ \\
\hline EDC1 & 3.51 & 2.67 & 498.4 & 354.4 & 142 & 133 \\
\hline EDC2 & 1.982 & 1.757 & 208.6 & 235.9 & 105 & 134 \\
\hline EDC3 & 1.830 & 1.796 & 200.4 & 186.8 & 110 & 104 \\
\hline EDC4 & 2.694 & 2.588 & 165.6 & 151.2 & 62 & 53 \\
\hline EDC5 & 3.80 & 3.55 & 177.4 & 166.5 & 47 & 47 \\
\hline EDC6 & 0.63 & 0.61 & 19.58 & 18.09 & 31 & 30 \\
\hline EDC7 & 6.582 & 5.673 & 867.1 & 765.3 & 132 & 135 \\
\hline EDC8 & 12.859 & 4.48 & 1485.3 & 747.4 & 116 & 167 \\
\hline EDC9 & 1.28 & 0.81 & 173.6 & 294.9 & 136 & 364 \\
\hline
\end{tabular}


Figures

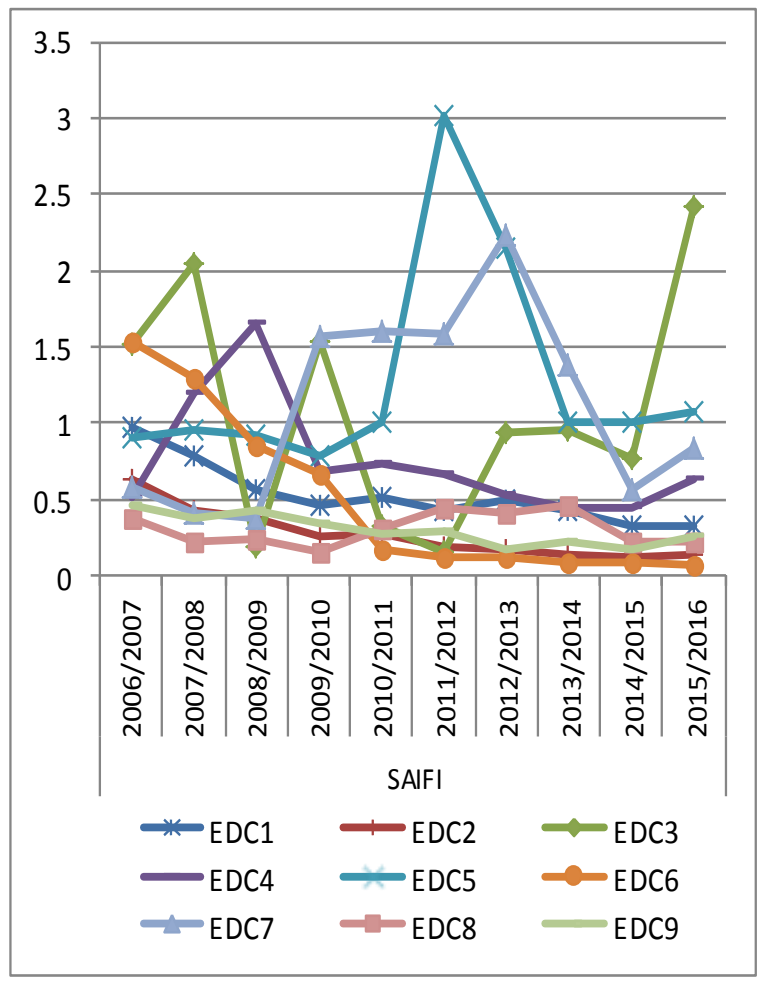

Fig.1. Historical EDCs system wide annual SAIFI matrices

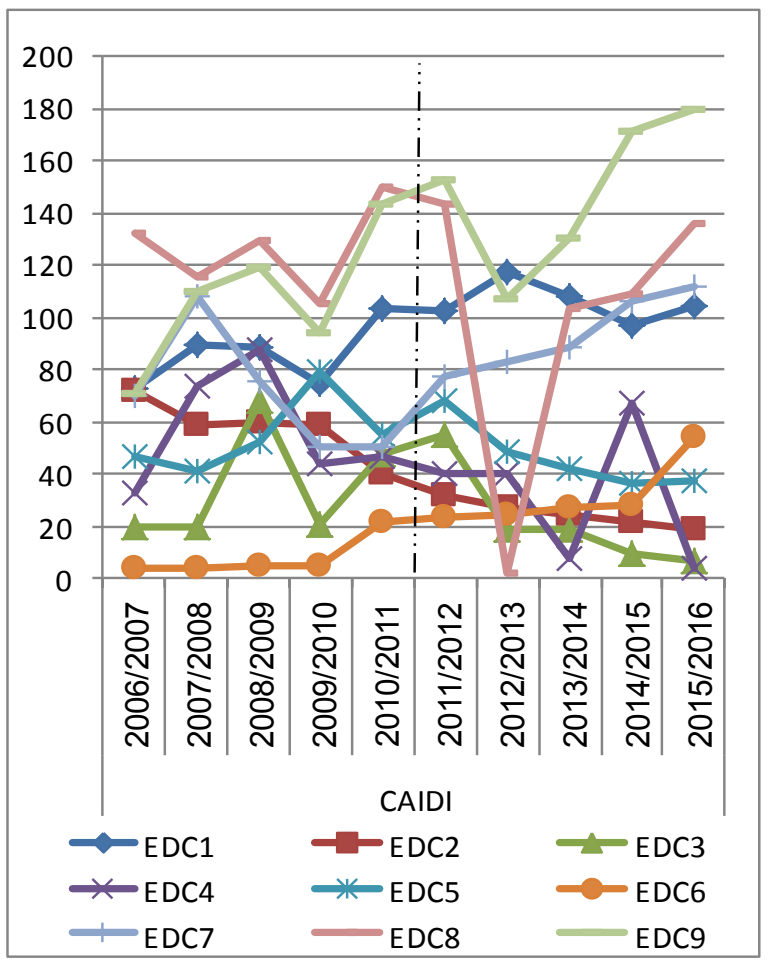

Fig.3. Historical EDCs system wide annual CAIDI matrices

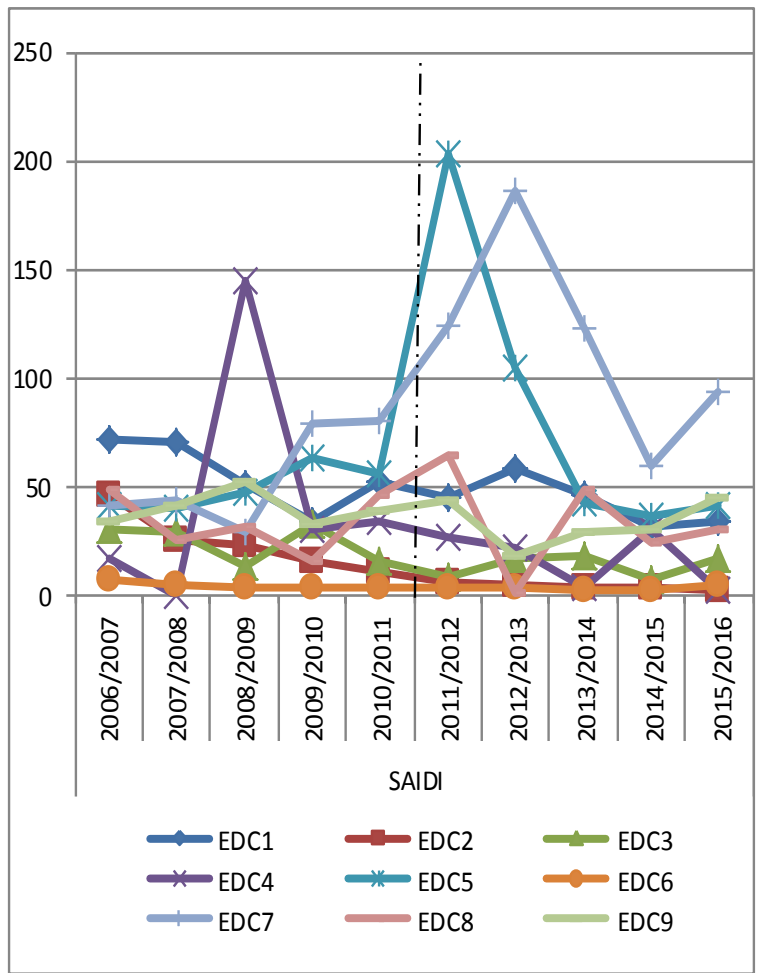

Fig.2. Historical EDCs system wide annual SAIDI matrices

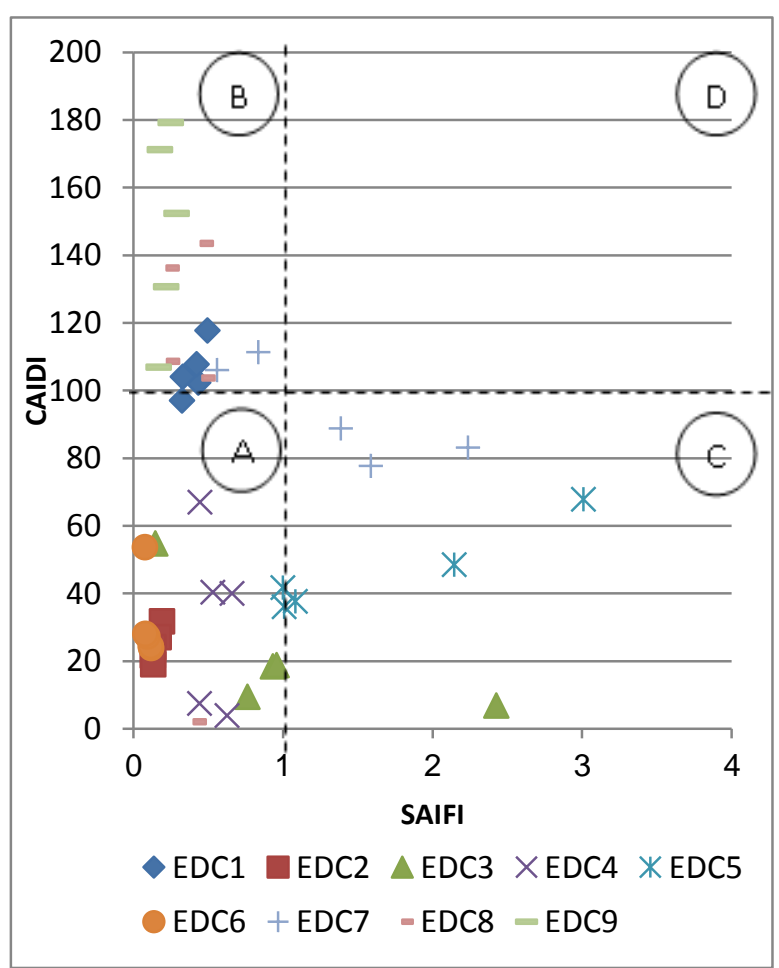

Fig.4. CAIDI versus SAIFI 


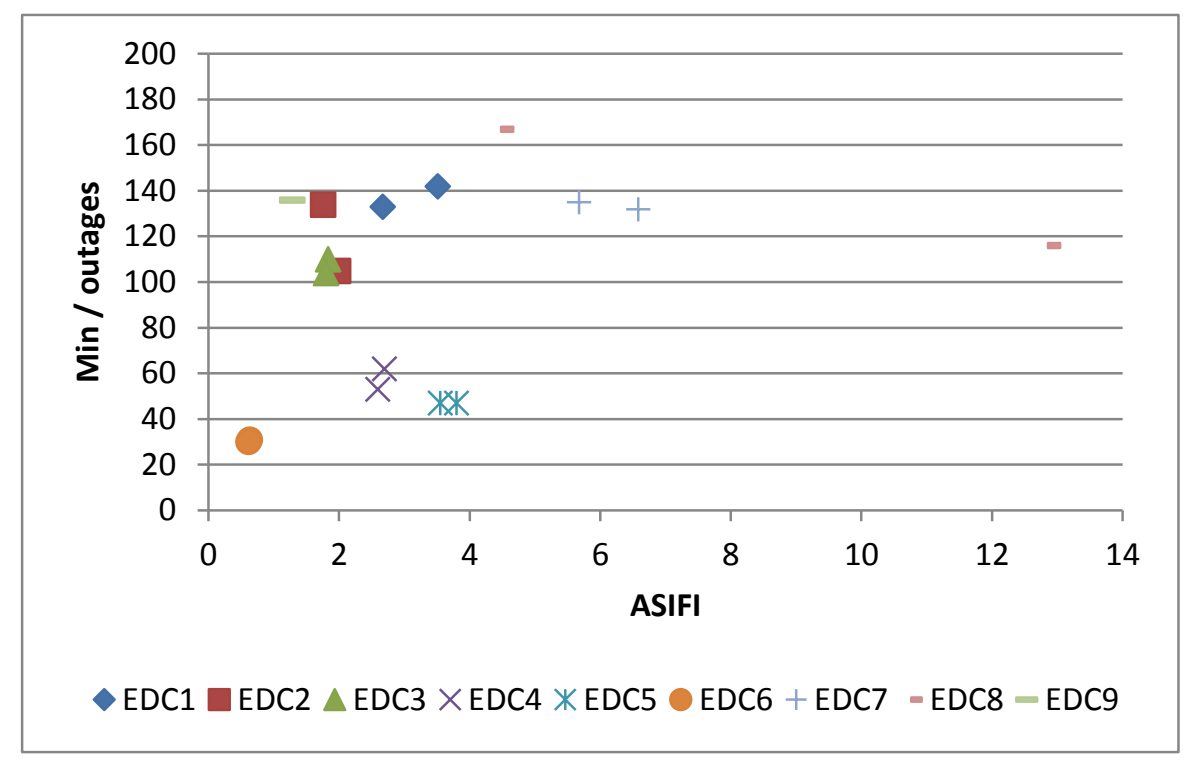

Fig.5. ASIFI versus min outages

\section{Reference}

[1] IEEE Power Engineering Society, IEEE Std. 1366: 2012 IEEE Guide for Electric Power Distribution Reliability Indices.

[2] CEER Benchmarking Report 5.2 - on the Continuity of Electricity Supply, Ref: C14-EQS-62-03,2015.

[3] Hafez El-Salmawy, Kamelia Youssef, Shereen Abdulla et al., " Reliability Indices: The identification of guideline values for electric networks in Egypt", Medpower 2010, Agia Napa Cyprus.

[4] Hafez El-Salmawy, Kamelia Youssef, Shereen Abdulla et al.," Indices of supply benchmarking EDCs in Egypt", MEPCON 2009, Assuit, Egypt.

[5] Hafez El-Salmawy, Kamelia Youssef, Shereen Abdulla et al.," Tracking the Reliability indicators In Electric Power System", MEPCON 2010, Cairo, Egypt.

[6] Hafez El-Salmawy, Kamelia Youssef, Shereen Abdulla et al.," Elementary Evaluation of Reliability Indices for Power System in Egypt", Cired 2011, Frankfort, Germany.

[7] Hafez El-Salmawy, Kamelia Youssef, Salma Hussein et al.," Power Quality: reliability indices for transmission system in Egypt", JNCN 2010, Amman, Jordan.

[8] Hafez El-Salmawy, Kamelia Youssef, Mohamed Abdel Rahman et al.," Extreme Events and Reliability Performance Indicators correlation for Distribution Utilities", Cired June 2013 ,Stockholm Sweden.

[9] Hafez El-Salmawy, Kamelia Youssef, Hatem Waheed et al.,"Key Performance Indices For EDCs in Egypt", MEPCON 2013,Cairo,Egypt.

[10] Hafez El-Salmawy, Kamelia Youssef, Shereen Abdulla et al.," Continuity of Supply Indicators for Egypt's Transmission Network", MEPCON 2012, ALEX, Egypt.

[11] Hatem Waheed, Kamelia Youssef, Eman Ahmed et al." Development of Reliability Indices for Electric Distribution Network in Egypt", ICNTSE, Alexandria, 2016. 